\title{
Thread as a substrate for low-cost point-of-care diagnostics
}

\author{
X. Li, D. Ballerini, J. Tian \&W. Shen \\ Australian Pulp and Paper Institute, \\ Department of Chemical Engineering, Monash University, Australia
}

\begin{abstract}
This study presents new applications of thread-based microfluidic systems in chemical and biochemical diagnostics. Our recent reports have demonstrated the capability of fabricating low-cost microfluidic devices using ubiquitous multifilament materials such as threads. The gaps between fibres in threads provide capillary wicking channels for liquid transport; therefore, liquid can penetrate along threads without the need of external forces. The new threadbased system, combined with the cheap dosing tools, is capable of easily and rapidly semi-quantifying the concentration of analytes in human body fluids. The utility of this system is further extended for the rapid and easy blood grouping with only small amount of whole blood ( $\sim 2 \mu \mathrm{L}$ for ABO blood typing), which is an essential test before blood transfusion. These low-cost and portable microfluidic devices are easy to fabricate, simple to use, and do not require powers such as electricity; thus providing a desirable analytical platform for point-of-care applications.
\end{abstract}

Keywords: low-cost diagnostics, microfluidic, point-of-care, thread-based.

\section{Introduction}

Modern microfluidic systems have been designed to carry out complicated analytical measurements for a range of applications [1]. However, such sophisticated microfluidic systems are usually expensive and the users always need to be trained to acquire the operation skills, thus restricting the practicability of these systems. The increasing needs of low-cost microfluidic devices which are readily accessible to average users, especially those people from under-developed areas or remote regions compel the development of new 
concepts and designs of inexpensive and easy-to-use microfluidic devices [2]. Paper-based microfluidic device, as a novel concept, was firstly proposed in 2007, then a plenty of subsequent studies from several research groups further developed this concept, either in the fabrication methods [3-7] or in the new applications [7-9]. Most recently, our group reported that cotton thread is also an ideal material to create microfluidic systems [10].

As an ancient and widely used material, cotton thread is cheap and can be manufactured in almost all areas of the world, even in the under-developed areas. Natural cotton fibres are hydrophobic because of the wax on the fibre surface [11]. However, with appropriate treatment (e.g. plasma), cotton thread can be made hydrophilic, and the gaps between thread fibres provide the capillary channels for liquids transport along thread without external pumps or driving forces. Besides, cotton thread is white and, therefore, presents a suitable background for colour expression for the colorimetric assay. All these properties make thread a suitable material to fabricate low-cost microfluidic thread-based analytical devices ( $\mu \mathrm{TADs}$ ) for biomedical or environmental applications.

In this paper we showed the surface modification of cotton thread using plasma for building $\mu$ TADs and developed a new method to measure the surface energy of thread. Ink jet ink solution was used to analyze liquid penetration and colour expression properties of hydrophilic threads. We reported for the first time that $\mu$ TADs can measure analyte concentration semi-quantitatively just with the cheap and simple sample dosing tools (e.g. sewing needle). This innovation avoided the use of expensive and precise quantitative sampling tools (e.g. micropipette), which made $\mu \mathrm{TADs}$ more suitable for field use under very limited conditions. Nitrite $\left(\mathrm{NO}_{2}^{-}\right)$, as an important biomarker indicating human health [12], was used as an example analyte to evaluate this analytical capability of $\mu$ TADs.

Another application of threads in point-of-care diagnostics presented is the identification of blood groups by virtue of separating agglutinated red blood cells (RBCs) from serum based on the sensitivity of the flow resistance of large particles in narrow capillary channels within threads. This new application has high diagnostic value for that the correct grouping of human blood is imperative before blood transfusion and it had been reported that without ABO compatibility testing, around one-third of unscreened blood transfusions would be expected to cause a haemolytic reaction [13].

All those new applications and advantages of $\mu$ TADs showed that this new system is simple and robust enough for the end users to perform basic healthcare sensing without special training. MicroTADs are portable, disposable, can be easily preserved and maintain a low cost of construction, making them attractive for diagnostics and point of care tests especially in remote and developing regions. 


\section{Materials and methods}

\subsection{Source of thread}

The raw (unbleached) cotton thread and polyester thread were obtained from the School of Fashion and Textiles, RMIT University, Melbourne, Australia. Our prior work showed that the raw cotton thread is hydrophobic to water because of the wax on the fibre surface. Plasma treatment can make thread hydrophilic, enabling liquid penetration along it.

\subsection{Thread surface analysis before and after plasma treatment}

An XPS analysis with an AXIS HIS spectrometer (Kratos) and monochromatized $\mathrm{Al} \mathrm{K} \alpha$ radiation was used to evaluate the surface modification of cotton thread with plasma. Thread samples were wound around a sample holder to form a closely packed mesh providing the necessary area $(\sim 2 \mathrm{~mm}$ in diameter) for the X-ray beam. Whatman no. 1 filter paper was used as the cellulose control. The photoelectron emission angle was $90^{\circ}$ with respect to the sample surface, corresponding to a maximum sampling depth of ca. $10 \mathrm{~nm}$.

\subsection{Preparation of test solutions}

Water (Millipore, $18 \mathrm{M} \Omega$ ) was used for making all solutions in this study. The commercial Canon ink jet magenta ink (http://www.canon.com.au/) was defined as the $100 \%$ ink solution; and it was diluted with water to obtain the $25 \%, 6.25 \%$ and $1.56 \%$ ink solutions for the colour display experiment on the hydrophilic thread.

The colorimetric detection of $\mathrm{NO}_{2}^{-}$is based on the principle of the Griess reaction [14]. The stock solution of $\mathrm{NO}_{2}^{-}(10.0 \mathrm{mmol} / \mathrm{L})$ was prepared by dissolving $69.0 \mathrm{mg}$ sodium nitrite $(\geq 99 \%)$ in $100 \mathrm{~mL}$ water. Then this stock solution was diluted with water to get serially diluted $\mathrm{NO}_{2}^{-}$standard solutions with the concentrations of $1000,500,250,125$ and $0 \mu \mathrm{mol} / \mathrm{L}$. The indicator solution for $\mathrm{NO}_{2}^{-}$contains $50 \mathrm{mmol} / \mathrm{L}$ sulfanilamide $(\geq 99 \%), 330 \mathrm{mmol} / \mathrm{L}$ citric acid ( $\geq 99.5 \%$ ), and $10 \mathrm{mmol} / \mathrm{L} \mathrm{N}$-(1-naphthyl) ethylenediamine ( $\geq 98 \%)$ [12]. All of the chemicals were purchased from Sigma-Aldrich.

Epiclone $^{\mathrm{TM}}$ anti-A, anti-B and anti-D monoclonal grouping reagents were obtained from CSL Australia. Blood samples (A+ and O-) were sourced from donors of known blood type, with samples being drawn by a trained nurse, and stored in Vacutainer ${ }^{\circledR}$ test tubes containing lithium-heparin anticoagulant. All blood samples were stored at $4^{\circ} \mathrm{C}$, and used within 5 days of withdrawal.

\subsection{Preparation of $\mu$ TADs for semi-quantitative detection of $\mathrm{NO}_{2}^{-}$}

Threads were used with polymer film to fabricate $\mu$ TADs by simply sewing the hydrophilic cotton thread through the film; colorimetric assays can be performed on threads. The white solid line (Figure 1) represents the stitches on the front side of the polymer film, while the dashed lines are the stitches on the back side. 


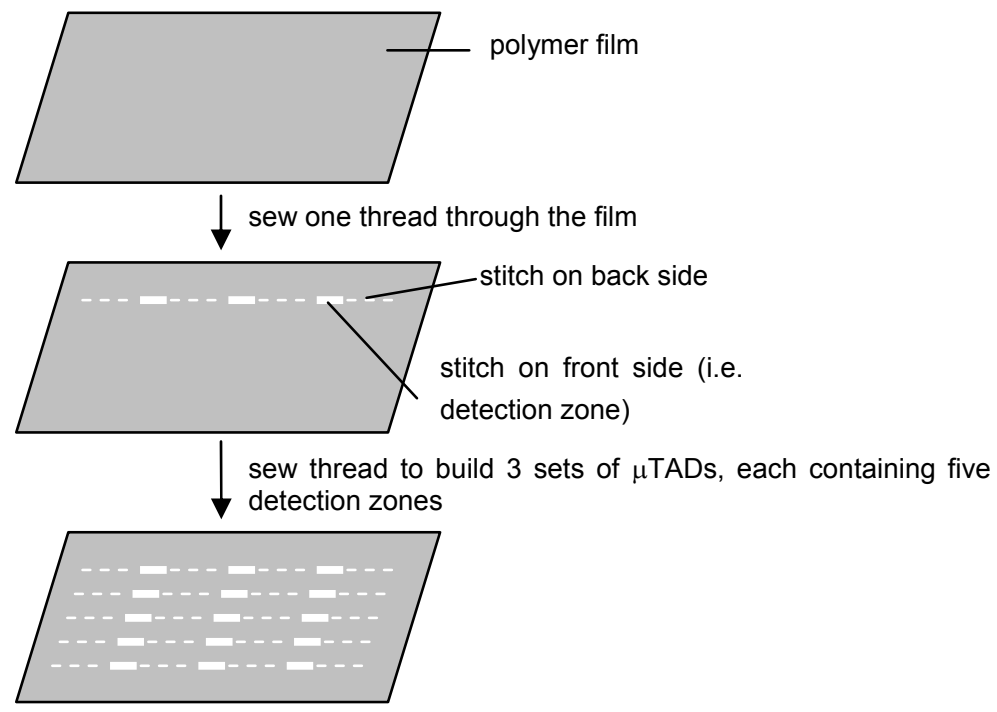

Figure 1: $\quad$ The scheme of the fabrication of $\mu$ TADs.

Each front side stitch $(\sim 3 \mathrm{~mm})$ was used as a detection zone of $\mu$ TADs. To quantify the assay results, $\mu$ TADs were scanned using a desktop scanner (Epson Perfection 2450 PHOTO, colour photo setting, $1200 \mathrm{dpi}$ ) and the images were analyzed using ImageJ 1.41o. For any selected area (i.e. detection zone), ImageJ calculated the average gray value within the selection, and background-corrected data was obtained by subtracting this value from the average intensity of the detection zone containing blank control solution $\left(0 \mu \mathrm{mol} / \mathrm{L} \mathrm{NO}_{2}^{-}\right)$, thereby showing the semi-quantitative results which are correlated with the concentration of the sample solutions.

\subsection{Preparation of thread-based platform for blood grouping}

Polyester threads were treated with antibody via soaking in the grouping reagent followed by blotting with standard blotting paper (Drink Coster Blotting, 280 $\mathrm{g} \cdot \mathrm{m}^{-2}$ ) to remove any surplus of antibody solution from the thread. After drying under fume hood for $10 \mathrm{~min}$, coated threads were ready to be used for $\mathrm{ABO}$ and $\mathrm{Rh}$ blood grouping.

\section{Results and discussion}

\subsection{Hydrophobic-hydrophilic contrast of untreated and plasma-treated threads}

Nisbet et al. have shown that the polar liquid penetration into a porous material occurred when the surface tension $(\gamma)$ of the liquid was lowered to a critical value 
and this value could be considered as the approximate surface free energy of the porous material [15]. Based on this principle, a series of isopropanol (IPA)-water solutions were prepared with different IPA mass percentages of 16, 18, 20, 22, and $24 \%$ (surface tension values were summarized in Table 1 [16]), then the surface free energy of natural cotton thread was measured by depositing a drop of IPA-water solution of different concentrations on thread and observing the drop penetration behaviour (i.e. the liquid forms a bead on thread surface or penetrates into thread). Table 1 shows that liquids of surface tension higher than $32.4 \mathrm{mN} / \mathrm{m}$ cannot penetrate along the thread, while liquids of surface tension lower than $31.2 \mathrm{mN} / \mathrm{m}$ can penetrate into thread. That means the surface free energy of the untreated cotton thread was between 31.2 and $32.4 \mathrm{~mJ} / \mathrm{m}^{2}$.

Table 1: $\quad$ Penetration behaviour of IPA + water on cotton thread.

\begin{tabular}{c|c|c}
\hline Isopropanol mass \% & Surface tension $\left(\gamma / \mathrm{mN}^{-1} \mathrm{~m}^{-1}\right)$ at $20^{\circ} \mathrm{C}$ & Penetration on cotton thread (Yes/No) \\
\hline 16 & 34.1 & No \\
18 & 32.4 & No \\
20 & 31.2 & Yes \\
22 & 29.9 & Yes \\
24 & 29.2 & Yes \\
\hline
\end{tabular}

Though natural cotton thread is hydrophobic to water, the fibre surface can be modified into hydrophilic with certain treatment (e.g. plasma in this work). The plasma-treated thread was strongly hydrophilic and easily wettable by water. Such difference in thread's wettability before and after treatment is referred as the hydrophobic-hydrophilic contrast. Figure 2 shows the XPS analysis of the surface chemistry of cotton threads before and after treatment to reveal changes in the surface atomic concentrations of $\mathrm{C}$ and $\mathrm{O}$.

Plasma treatment may have two effects on thread surface modification. First, the wax was partially removed, thus exposing the underlying cellulose, which was indicated by the increase in surface concentration of $\mathrm{C}-\mathrm{O}(\mathrm{BE}=286.8 \mathrm{eV})$. Second, thread surface was substantially oxidized, which was revealed by the increase in surface concentrations of $\mathrm{O}-\mathrm{C}=\mathrm{O}, \mathrm{O}-\mathrm{C}-\mathrm{O}$ and $\mathrm{C}=\mathrm{O}(\mathrm{BE}=289.2$, 288.3 and $288.3 \mathrm{eV}$, respectively). These changes increased the surface oxygen concentration and the surface polarity of the thread, making the plasma-treated thread hydrophilic.

\subsection{Liquids penetration along hydrophilic thread and colour display on thread}

Liquid penetration along the hydrophilic thread was investigated to understand the relationship between the volume of liquid introduced onto thread and the liquid penetration distance along thread. Four pieces of threads were fixed in parallel onto a supporting film. Magenta ink solutions of different volume $(0.1$, $0.2,0.4,0.8 \mu \mathrm{L}$ ) were introduced onto each thread using a micropipette (Eppendorf Research $^{\circledR} 0.1-2.5 \mu \mathrm{L}$ ) and penetrated along thread with different 
distance (Figure 3a). A calibration curve (Figure 3b) from six independent measurements shows that the liquid penetration length almost linearly correlates to the liquid volume (when volume $\leq 0.4 \mu \mathrm{L}$ ). This correlation became curved as the liquid volume increased beyond $0.4 \mu \mathrm{L}$. The near-linear section of the calibration curve suggests that the ink "concentration" on the thread (i.e. the amount of dye molecules on each unit length of thread) is constant and is independent on the ink volume. This indicates that a semi-quantitative analysis can still be performed on $\mu \mathrm{TADs}$ even without precise liquid-handling instrument (as long as sample volume $\leq 0.4 \mu \mathrm{L}$ ).

To further prove this prediction, three parallel sets of $\mu$ TADs were fabricated and each $\mu \mathrm{TAD}$ has four detection zones vertically arranged from top to bottom (Figure $4 \mathrm{a})$. Ink solutions of $0.1 \mu \mathrm{L}$ of different concentrations $(1.56 \%, 6.25 \%$, $25 \%$, and $100 \%$ ) were added to $\mu$ TAD 1 (the left set). Similarly, ink solutions of $0.2 \mu \mathrm{L}$ were added to $\mu \mathrm{TAD} 2$ (the middle set) and those of $0.4 \mu \mathrm{L}$ were added to $\mu$ TAD 3 (the right set). The adding sequence is shown in Figure 4a. The penetration length of $0.1 \mu \mathrm{L}$ ink solution is about $5 \mathrm{~mm}$; for ink solutions of

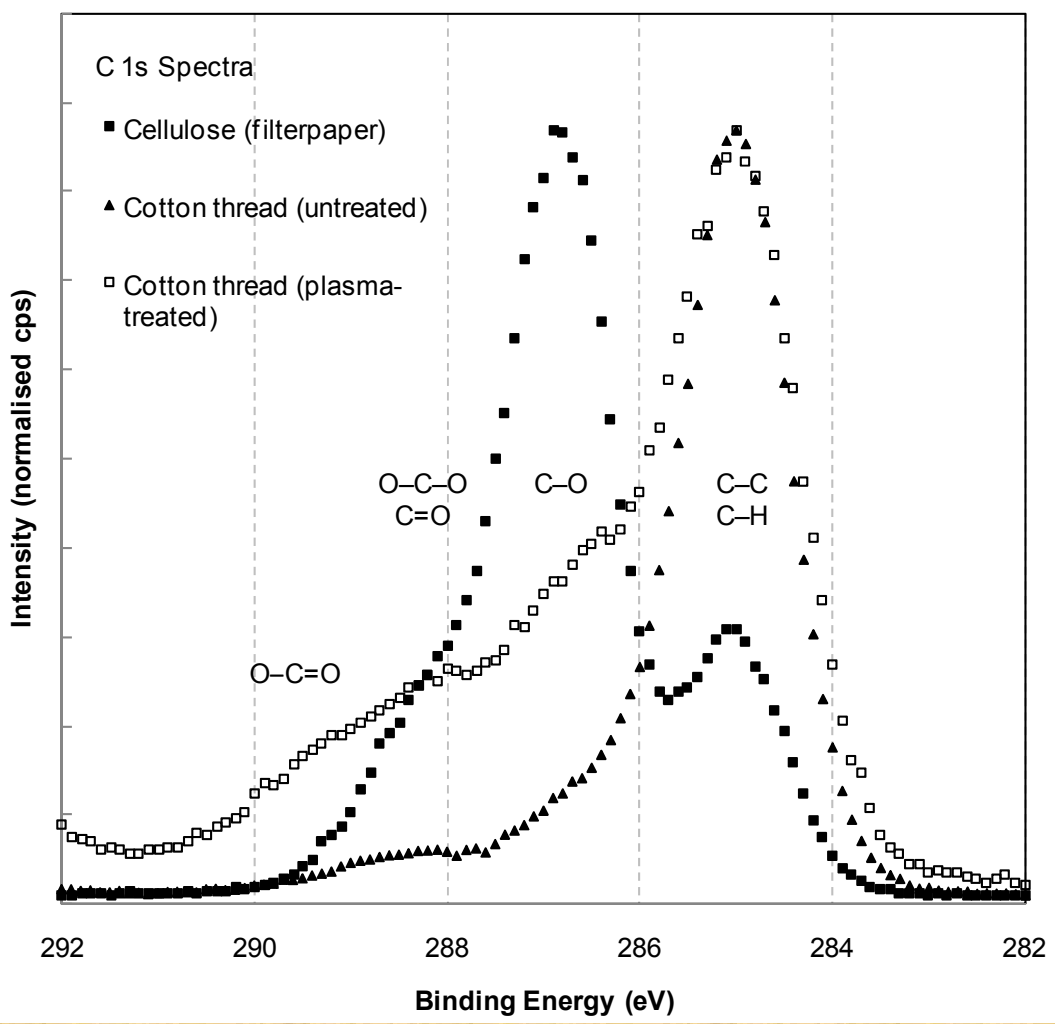

Figure 2: XPS results of the untreated (hydrophobic) thread and plasmatreated (hydrophilic) thread. Filter paper is used as the control sample for cellulose. 
a

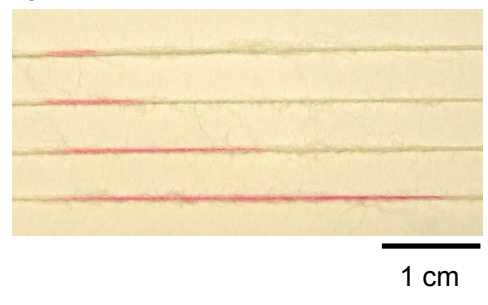

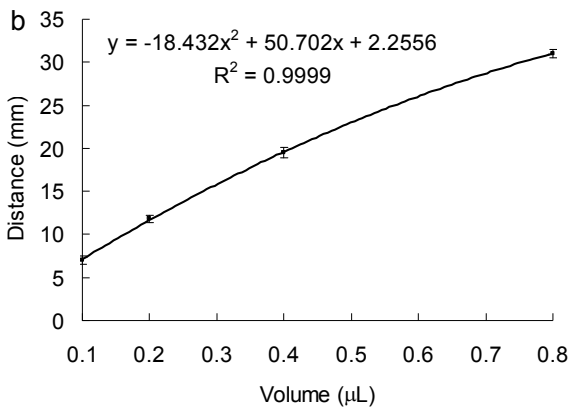

Figure 3: (a) Ink penetration on hydrophilic thread. Ink solutions of different amount $(0.1,0.2,0.4,0.8 \mu \mathrm{L})$ are introduced onto four pieces of thread from top to bottom; (b) the average ink penetration length obtained from six repeated tests using a Vernier caliper.

a

$0.1 \mu \mathrm{L} \quad 0.2 \mu \mathrm{L}$

$0.4 \mu \mathrm{L}$

$1.56 \%$

$6.25 \%$

$25 \%$

$100 \%$ b

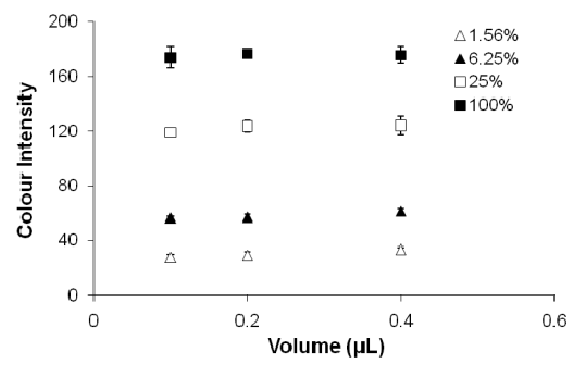

$5 \mathrm{~mm}$

Figure 4: (a) Colour display of different ink solutions on 3 sets of $\mu$ TADs. The volume of ink is $0.1,0.2$ and $0.4 \mu \mathrm{L}$, respectively (from left to right); the concentration of ink is $1.56 \%, 6.25 \%, 25 \%$ and $100 \%$, respectively (from top to bottom); (b) The average \pm s.d. colour intensity of each observation zone is obtained from six independent tests.

larger volume, the extra amount of solution penetrates along thread to the back side of polymer film and cannot be seen. For each $\mu \mathrm{TAD}$, the colour of ink solution varies from weak to strong as ink concentration increases. However, the colour of ink solution of same concentration and different volume has negligible variation which is proved by colour intensity measurement. For each detection zone, a fixed rectangle area is set to encompass the zone and the mean colour intensity of the selected area is analyzed. Six independent measurements performed provide the average \pm standard deviation (s.d.) data of colour intensity (Figure 4b). The results show that the average colour intensity is consistent for ink solution of different volume and same concentration; the small standard deviation indicates that thread is a good substrate for colour display. 


\subsection{Semi-quantitative measurement of $\mathrm{NO}_{2}^{-}$using $\mu$ TADs and cheap liquid dosing tool}

Since the colour intensity of sample solutions spotted on $\mu$ TADs is not affected by the sample volume (volume $\leq 0.4 \mu \mathrm{L}$ ), semi-quantitative assays can be performed even if high precision liquid-handling instrument is unavailable. A household sewing needle was used in this work as a simple means to spot liquids. We found that by rapidly dipping the needle eye into liquids, it can pick up liquid of $0.22 \pm 0.04 \mu \mathrm{L}$ (average \pm s.d., 12 repeats) after moving out from the solution. This small volume variation does not cause a significant colorimetric error for the reasons discussed above. Water and 70\% ethanol were used to wash and disinfect the needle eye for repeated use.
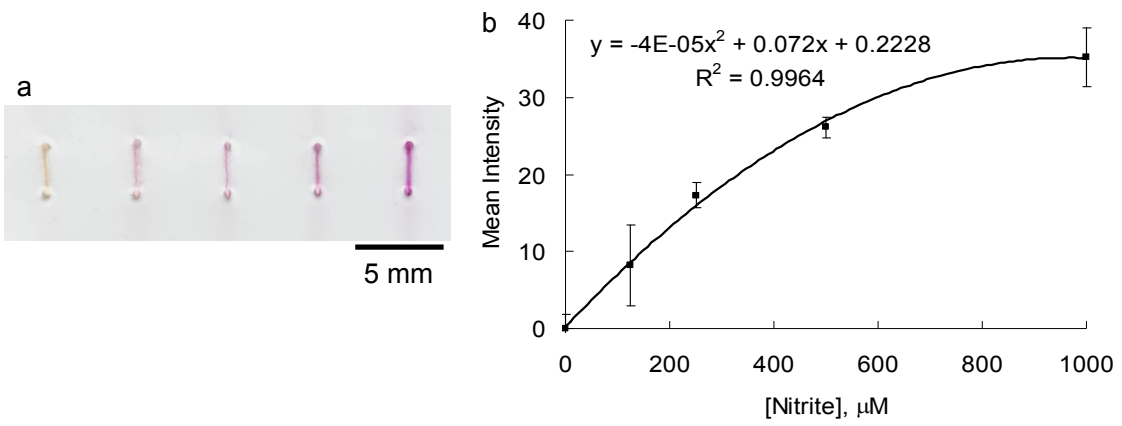

Figure 5: (a) $\mathrm{NO}_{2}^{-}$sample solutions of different concentration $(0,125,250$, 500 , and $1000 \mu \mathrm{M}$ ) were added using needle eye onto $\mu \mathrm{TAD}$ from left to right. $\mathrm{NO}_{2}^{-}$triggered different colour changes on each detection zone; (b) semi-quantitative results of the relationship between $\mathrm{NO}_{2}^{-}$concentration and colour intensity $(n=6)$.

Nitrite $\left(\mathrm{NO}_{2}^{-}\right)$was used as an analyte to demonstrate the feasibility of using $\mu \mathrm{TADs}$ and cheap sample dosing tool to perform semi-quantitative analysis. The hydrophilic thread was soaked in $\mathrm{NO}_{2}{ }^{-}$indicator solution and then dried in oven $\left(60^{\circ} \mathrm{C}\right)$ for $5 \mathrm{~min}$; the indicator-treated thread was sewn onto a polymer film to fabricate $\mu \mathrm{TAD}$ (procedure in Figure 1) containing five detection zones for colorimetric measurement. Five standard $\mathrm{NO}_{2}^{-}$solutions $(0,125,250,500$ and $1000 \mu \mathrm{M}$ ) were introduced onto each detection zone (from left to right) using the needle eye and caused different colour development from colourless to pink of different shades (Figure 5a). The $\mathrm{NO}_{2}{ }^{-}$concentration calibration curve can be obtained through colorimetric analysis of those detection zones with the average \pm s.d. data of colour intensity from six independent tests on six $\mu$ TADs. Therefore, $\mu \mathrm{TADs}$, together with cheap liquid-handling tool, can be used as new low-cost, low-volume and easy-to-use analytical platform for health or environmental care in developing and remote regions with limited resources; and the users only need to get basic training for utilizing them. 


\subsection{Blood grouping using thread-based platform}

To investigate the feasibility of using antibody-treated threads as blood grouping platform, three types of thread (thread treated with anti-A, anti-B and anti-D, respectively) were immobilized in folded polypropylene films with lodging slits to aid testing, and a micropipette (Eppendorf research ${ }^{\circledR} 0.1-2.5 \mu \mathrm{L}$ ) was used to dose whole-blood sample of $1 \mu \mathrm{L}$ onto each thread. If red blood cells (RBCs) have haemagglutination reaction with the antibody deposited on thread, a separation band can be seen on thread. The separation distance is around $3 \mathrm{~mm}$ (similar length to a standard "stitch" found on a shirt cuff), and is therefore easily visible by unaided human eye. Typically, results became observable within 1 min of sample dosing, making the test quite rapid compared to some existing techniques. Identifying a person's $\mathrm{ABO}$ and $\mathrm{Rh}$ blood type requires three tests, two for $\mathrm{A}$ and $\mathrm{B}$ antigens to determine $\mathrm{ABO}$ grouping, and a further test for $\mathrm{D}$ antigen to verify $\mathrm{Rh}$ grouping. A results-matrix (Table 2) aids in the interpretation of results.

Table 2: $\quad$ Results-matrix for blood grouping interpretation. Tick indicates a positive result (separation occurs); crosses indicate a negative result (no separation).

\begin{tabular}{|r|c|c|cc|cc|c|c|}
\hline Anti - A & $\checkmark$ & $\checkmark$ & $\checkmark$ & $\checkmark$ & $\times$ & $\times$ & $\times$ & $\times$ \\
\cline { 2 - 9 } Anti - B & $\checkmark$ & $\checkmark$ & $\times$ & $\times$ & $\checkmark$ & $\checkmark$ & $\times$ & $\times$ \\
\hline Anti -D & $\checkmark$ & $\times$ & $\checkmark$ & $\times$ & $\checkmark$ & $\times$ & $\checkmark$ & $\times$ \\
\hline & AB+ & AB- & A+ & A- & B+ & B- & O+ & O- \\
\hline
\end{tabular}

Identifying the agglutination of RBCs using thread has a unique advantage: large and discrete particles in a suspension system undergo a phase separation from the continuous liquid phase in the narrow capillary channels. As wholeblood sample penetrates through the inter-fibre gaps of the antibody-treated threads, blood serum will dissolve the antibody molecules on the fibre surface. If antibodies bond to the specific binding sites on the antigens of adjacent RBCs (i.e. the haemagglutination reaction happens), the aggregation of RBCs by the antibody molecules leads to the formation of significantly larger particles that cannot be stably suspended in the serum phase. As the sample penetrates along thread, the size of the particles increases and the agglutinated RBCs cannot contribute to the capillary driving force required for the blood sample to continue penetrating forward. Instead, the agglutinated RBCs can only be carried by the serum to move forward. In this situation agglutinated RBCs will be gradually left behind from the serum penetration front. Furthermore, agglutinated RBCs in the inter-fibre channels may act as a "filter" which permits serum to pass, but prevents agglutinated RBCs from passing. This causes the separation of agglutinated RBCs from the serum phase and the separation is visually 
identifiable by the appearance of a pale pink coloured band between the agglutinated RBCs and dry antibody residue, as seen in Figure 6a, 6c. In the case where the deposited antibody does not react with the antigen of RBCs, no separation is visually detectable (as seen for type O- blood in Figure 6d-f).

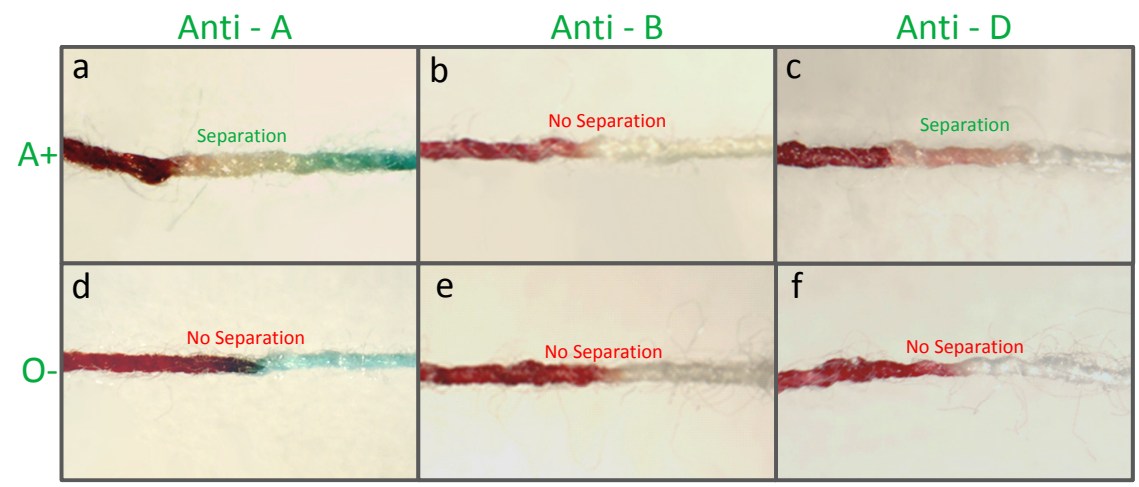

Figure 6: $\quad$ Proof of concept tests with samples of whole blood of type A+ and O- on antibody-treated polyester threads. Columns show results on threads treated with anti-A, anti-B and anti-D from left to right; rows show the results for the different blood types as labelled on the left.

\subsection{Storage stability of the devices}

Referring to the ultimate practical application of the $\mu$ TADs, it is important that these devices must be stable for a certain period (e.g. one month or longer) to become commercialized products for consumers. In this study, $\mu$ TADs fabricated for semi-quantitative $\mathrm{NO}_{2}^{-}$detection were stored in a light-tight container in a desiccator at room temperature. The performance of $\mu$ TADs showed no deterioration after 6 weeks, indicating that these low-cost and easy-to-use $\mu$ TADs have sufficient shelf life for practical use.

The efficacy of the thread-based platform for blood grouping was investigated after a 4 week storage period. Two sets of antibody-treated threads were stored in microtubes wrapped in foil; one at $4^{\circ} \mathrm{C}$ the other at ambient temperature. All tests showed the correct grouping, suggesting that the device could be stored for a moderate period of time without degrading if sealed in airtight and light-proof packaging.

\section{Conclusion}

Thread, a cheap and widely available material, was used in this study to fabricate low-cost microfluidic analytical devices; the analytical capabilities of these devices were studied in detail. The hydrophobic natural cotton threads can be surface modified using plasma treatment into hydrophilic threads, allowing the 
transport of aqueous liquids via capillary driving force. Threads as liquid transport channels do not rely on physical or chemical barriers, enabling $\mu$ TADs to transport low surface tension liquids such as organic solvents or oil, which may not be possible for some paper-based microfluidic channels. Threads are white and stainable, making them suitable for color display. Semi-quantitative tests of analytes can be rapidly and easily performed on $\mu$ TADs through colorimetric reaction using the non-precision household liquid handling items such as sewing needle which are also available in resource-limited regions. Based on this principle the concentration of $\mathrm{NO}_{2}^{-}$(a biomarker for indicating some human diseases) in aqueous samples has been successfully measured.

Thread was further used as a porous substrate for blood grouping in this work, which requires only whole blood of small amount (e.g. $\sim 2 \mu \mathrm{L}$ for $\mathrm{ABO}$ blood grouping) and eliminates the need for the end user to either handle other testing reagents or perform sample dilutions. Although only $A B O$ and $\mathrm{Rh} / \mathrm{D}$ blood groups were tested in this study, it is expected that the platform could easily be extended to identify other groups of interest that follow similar antibody/antigen interactions.

All these $\mu$ TADs and thread-based blood grouping system prototyped are simple and robust enough to be employed by the end user without assistance. They are portable, disposable, can be easily preserved and maintain a low cost of construction, making them attractive for diagnostics and point of care testing especially in remote and developing regions.

\section{Acknowledgements}

The authors would like to thank the kind blood donors: Mr. Scot Sharman, Mr. Henri Kröling; as well as Ms. Lisa Collison of the Monash University Health Service for collecting blood donations. The authors also thank Dr. Lijing Wang of the School of Fashion and Textiles, RMIT University, for kindly providing thread samples. The research scholarships of Monash University and the Department of Chemical Engineering are gratefully acknowledged. The Postgraduate Research Travel Grant from Monash Research Graduate School (MRGS) and the New Speakers Contest prize from Appita are also appreciatively acknowledged for supporting the prospective conference participation.

\section{References}

[1] West, J., Becker, M., Tombrink, S. \& Manz, A., Micro total analysis systems: latest achievements. Analytical Chemistry, 80(12), pp. 4403-4419, 2008.

[2] Zhao, W. \& van den Berg, A., Lab on paper. Lab on a Chip, 8(12), pp. 1988-1991, 2008.

[3] Martinez, A.W., Phillips, S.T., Butte, M.J. \& Whitesides, G.M., Patterned paper as a platform for inexpensive, low-volume, portable bioassays. Angewandte Chemie International Edition, 46(8), pp. 1318-1320, 2007. 
[4] Abe, K., Suzuki, K. \& Citterio, D., Inkjet-printed microfluidic multianalyte chemical sensing paper. Analytical Chemistry, 80(18), pp. 6928-6934, 2008.

[5] Li, X., Tian, J., Nguyen, T. \& Shen, W., Paper-based microfluidic devices by plasma treatment. Analytical Chemistry, 80(23), pp. 9131-9134, 2008.

[6] Li, X., Tian, J., Garnier, G. \& Shen, W., Fabrication of paper-based microfluidic sensors by printing. Colloids and Surfaces B: Biointerfaces, 76(2), pp. 564-570, 2010.

[7] Dungchai, W., Chailapakul, O. \& Henry, C.S., Electrochemical detection for paper-based microfluidics. Analytical Chemistry, 81(14), pp. 58215826, 2009.

[8] Hossain, S.M.Z., Luckham, R.E., McFadden, M.J. \& Brennan, J.D., Reagentless bidirectional lateral flow bioactive paper sensors for detection of pesticides in beverage and food samples. Analytical Chemistry, 81(21), pp. 9055-9064, 2009.

[9] Li, X., Tian, J. \& Shen, W., Quantitative biomarker assay with microfluidic paper-based analytical devices. Analytical and Bioanalytical Chemistry, 396(1), pp. 495-501, 2010.

[10] Li, X., Tian, J. \& Shen, W., Thread as a versatile material for low-cost microfluidic diagnostics. ACS Applied Materials \& Interfaces, 2(1), pp. 16, 2010.

[11] Wakelyn, P.J., Cotton. Kirk-Othmer Encyclopedia of Chemical Technology, John Wiley \& Sons, Inc., pp. 19-20, 2000.

[12] Blicharz, T.M., Rissin, D.M., Bowden, M., Hayman, R.B., DiCesare, C., Bhatia, J.S., Grand-Pierre, N., Siqueira, W.L., Helmerhorst, E.J., Loscalzo, J., Oppenheim, F.G. \& Walt, D.R., Use of colorimetric test strips for monitoring the effect of hemodialysis on salivary nitrite and uric acid in patients with end-stage renal disease: a proof of principle. Clinical Chemistry, 54(9), pp. 1473-1480, 2008.

[13] Daniels, G. \& Reid, M.E., Blood groups: the past 50 years. Transfusion, 50(2), pp. 281-289, 2010.

[14] Sun, J., Zhang, X., Broderick, M. \& Fein, H., Measurement of nitric oxide production in biological systems by using Griess reaction assay, Sensors, 3(8), pp. 276-284, 2003.

[15] Nisbet, D.R., Pattanawong, S., Ritchie, N.E., Shen, W., Finkelstein, D.I., Horne, M.K. \& Forsythe, J.S., Interaction of embryonic cortical neurons on nanofibrous scaffolds for neural tissue engineering. Journal of Neural Engineering, 4(2), pp. 35, 2007.

[16] Vazquez, G., Alvarez, E. \& Navaza, J.M., Surface tension of alcohol + water from 20 to $50{ }^{\circ} \mathrm{C}$. Journal of Chemical \& Engineering Data, 40(3), pp. 611-614, 1995. 